\title{
A NEW SPECIES OF CHOCOAN STURNIRA (CHIROPTERA: PHYLLOSTOMIDAE: STENODERMATINAE) FROM WESTERN ECUADOR AND COLOMBIA
}

\author{
TIMOTHY J. MCCARTHY \\ Scientific Preparator, Section of Mammals; \\ Carnegie Museum of Natural History, 5800 Baum Blvd., Pittsburgh, PA 15206-3706 (McCarthyT@CarnegieMNH.org) \\ Luis Albuja V. \\ Instituto de Ciencias Biológicas, Escuela Politécnica Nacional, Apartado 17-01-2759, Quito, Ecuador (lalbuja@server.epn.edu.ec) \\ Michael S. Alberico \\ Departamento de Biología, Universidad del Valle, Apartado 25360, Cali, Colombia [Deceased]
}

\begin{abstract}
A distinctive new species of sturnirine bat, Sturnira (Sturnira) koopmanhilli, new species (Chiroptera: Phyllostomidae: Stenodermatinae), is described from 49 specimens collected in the Pacific drainage of the western Andes, at 300-2000 m in western Ecuador and Colombia. This yellow-shouldered bat is endemic to this Chocoan region where it is known from nine localities in Ecuador (Esmeraldas and Chimborazo) and Colombia (Chocó, Valle del Cauca, and Nariño). This moderately large Sturnira species (forearm length, 48.1-52.4 mm) is compared with Sturnira mordax (Goodwin, 1938) (forearm length, 43.0-49.2) and Sturnira ludovici Anthony, 1924 (forearm length, 43.9-49.5). Salient characters that distinguish the new species from these two cogeners include a bicolored appearance of dorsal fur, recessed hypoglossal foramina, presence of a sulcus on posterior faces of upper canines, and teeth P2 > M2. Teeth I1 and i1 are protrudent and robust, which set the new species apart from other Sturnira species. Spacing between teeth P1-M3 and p1-m3 is present in the new bat and with the subgenus Corvira. The new species is placed in the subgenus Sturnira.
\end{abstract}

KeY Words: Sturnira, bat, new species, Chiroptera, Ecuador, Colombia, Chocó

\section{INTRODUCTION}

A region of high rainfall extends along the Pacific lowlands and mid-Andean slopes of western Ecuador and Colombia to extreme southeastern Panama. This area is referred to as the Chocó after the Colombian department where record rainfall supports tropical pluvial forest (Gentry 1982; Haffer 1967). As a biotic unit, the forests of the lowland and contiguous premontane habitats of the Chocó have been characterized as possessing the richest and most diverse flora in the world (Gentry 1982, 1986).

Overlaying this vegetative complexity, an incomplete assessment of the vertebrate fauna reveals a high incidence of species diversity and endemism centered in the Chocó biotic province (Handley 1966; Haffer 1967; Lynch 1971, 1980; Duellman 1979; Terborgh and Winter 1982).

Understanding the extent of endemism in the bat fauna of the Chocó has been an objective during field vertebrate inventories by personnel of the Instituto de Ciencias Biológicas, Escuela Politécnica Nacional, Ecuador, and the Departamento de Biología, Universidad del Valle, Colombia. Known Chocoan bat endemics include Balantiopteryx infusca, Choeroniscus periosus, Lonchophylla chocoana, L. orcesi, Rhinophylla alethina, Platyrrhinus chocoensis, and Molossops aequatorianus (Handley 1966; Ibañez 1980; Alberico and Velasco 1991; Albuja 1999; Alberico et al. 2000; McCarthy et al. 2000; Dávalos 2004; Albuja and Gardner 2005).

We add a distinctive new species of Sturnira (Chiroptera: Phyllostomidae: Stenodermatinae) to the
Chocoan fauna. Specimens of this new species from Colombia have been confused with S. mordax (Alberico 1994; McCarthy et al. 2005). A report of S. mordax in the region may have influenced Sánchez-Hernández et al. (2002) to report an unfamiliar specimen of S. ludovici from Ecuador as S. mordax (McCarthy et al. 2005). Consequently, our new species is compared with Sturnira mordax (Goodwin, 1938), which is endemic to mountainous Costa Rica and western Panama, and with Sturnira ludovici Anthony, 1924, a sympatric species in the Chocó.

\section{MATERIALS AND METHODS}

Thirty-three specimens from two sites in the provinces of Esmeraldas (1) and Chimborazo (1), Ecuador, and 16 specimens from seven Colombian localities in the departments of Chocó (3), Valle del Cauca (3), and Nariño (1) represent the Sturnira sp. nov. (Fig. 1; localities arranged north to south). Each locality on the map (Fig. 1) is indicated by a number, which is in the list of paratypes. The listing in the Specimens Examined section for S. mordax (Costa Rica, Panama) and S. ludovici (Ecuador) are arranged alphabetically by province, within which the localities are arranged north to south.

This description focuses principally on the pool of specimens of the new Sturnira and S. ludovici from the type locality. The external and cranial measurements were taken with calipers under magnification. Three length measure- 


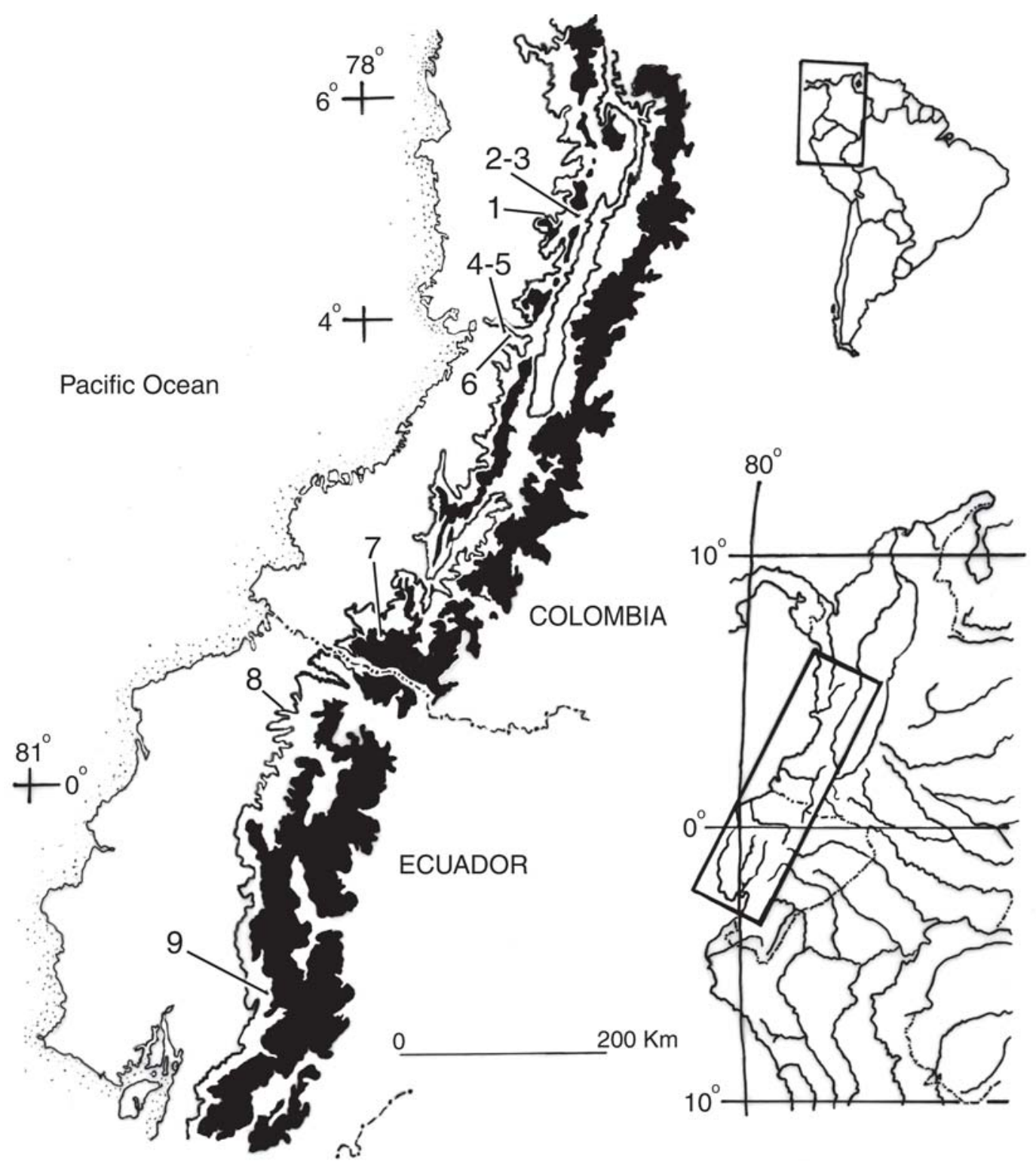

Fig. 1. - General map of western Ecuador and Colombia with the western Andean slope shaded above $2000 \mathrm{~m}$. Numbers 1-9 indicate collecting localities for the new species in the Chocó. The numbers correspond with those included in the text for the nine localities in the designation of paratypes.

ments (TL, HF, E) were taken with a metric ruler during specimen preparation in the field. Mass was determined with a spring scale. All linear measurements are in millimeters $(\mathrm{mm})$; mass is in grams $(\mathrm{g})$. We list the external and field measurements (Table 1; text) with the corresponding abbreviations: length of forearm (FA), distance from bent elbow and collapsed wing to farthest point on wrist including carpals; the lengths of the third and fifth metacarpals (MIII, MV) including the carpals; the first phalanx on the third (IIIPh1), fourth (IVPh1), and fifth (VPh1) fingers, and the second phalanx on third finger (IIIPh2) are measured end to end at bent joints. Wing measurements were taken from fluid-preserved speci- mens and dried study skins. The length of tibia (TB), distance from top of tibia with bent knee to base of juncture with calcar; total length (TL), dorsally, distance from tip of the last vertebra to nose tip; length of hindfoot (HF), distance along foot from anterior edge of calcar to most distant claw; length of ear (E), distance from ear notch to pinna tip.

The cranial measurements (Table 2 ; text) are described in McCarthy et al. (2005). We modify certain measurements (CM3, MM, CC) to include distance across tooth crowns instead of alveolar positions. Other measurements include: greatest length of skull (excludes incisors, GLS); zygomatic breadth (ZB); breadth of braincase (BB); mas- 
toidal breadth (MB); postorbital constriction (POC); depth of braincase (BD); length of palate (PL); postpalatal length (PPL); length of maxillary tooth row (CM3); breadth across upper molars (MM); breadth across upper canines (CC); length of upper canine (CL); length of mandible (from tip of mandibular symphysis, ML); height of coronoid process $(\mathrm{CPH})$.

Abbreviations for incisor (I, i), canine $(\mathrm{C}, \mathrm{c})$, premolar $(\mathrm{P}, \mathrm{p})$, and molar $(\mathrm{M}, \mathrm{m})$ teeth are capitalized when in the upper tooth row and in lower case when in the lower tooth row. Numbered teeth (e.g., I2, p1, M3) refer to position in tooth rows. Relative size index of premolars and molars was compared among the three species of Sturnira. This determination was based on drawings of the occlusal surface areas, which were scored on graph paper to the same scale, utilizing a binocular scope with camera lucida attachment. Size between different teeth is expressed as approximate percentage. The orientation of the soft palatal ridges in relation to alignment with the maxillary tooth rows was examined. Unprepared skulls of S. mordax (USNM 520832, 520835, 520837, 520838, 541345), S. ludovici (CM 112837-112840) and the new Sturnira (EPN 2270, 2287, 2289) were studied.

We follow Hershkovitz (1971) for our use of dental cusp terminology and orientation. This is for descriptive purposes and does not imply acceptance of homologies.

Institutional abbreviations. - AMNH-Department of Mammalogy, American Museum of Natural History, New York, New York; CMSection of Mammals, Carnegie Museum of Natural History, Pittsburgh, Pennsylvania; EPN-Instituto de Ciencias Biológicas, Escuela Politécnica Nacional, Quito, Ecuador; FMNH—Division of Mammals, Field Museum of Natural History, Chicago, Illinois; MCZ - Museum of Comparative Zoology, Harvard University, Cambridge, Massachusetts; MNHN-Laboratoire Mammifères et Oiseaux, Muséum National d'Histoire Natural, Paris, France; UMMZ - Division of Mammals, University of Michigan Museum of Zoology, Ann Arbor, Michigan; USNM - Division of Mammals, National Museum of Natural History, Washington, D.C.; UV-Departamento de Biología, Universidad del Valle, Cali, Colombia.

\section{SYSTEMATIC ZOOLOGY}

Order Chiroptera Blumenbach, 1779

Family Phyllostomidae Gray, 1825

Subfamily Stenodermatinae Gervais, 1856

Genus Sturnira Gray, 1842

\section{Sturnira (Sturnira) koopmanhilli, new species Figures 3-9, Tables 1-3}

Holotype.-Adult male, EPN 9770, collected 26 November 1991 by I. Manzano and T.J. McCarthy, field number TJMc 7553. The specimen was prepared as a dry skin and skull; the carcass is preserved in alcohol.

Type locality.-Los Pambiles, confluence of unnamed river with Río las Piedras, Reserva Ecológica Cotacachi-Cayapas, Provincia de Esmeraldas, Ecuador, $00^{\circ} 32^{\prime} \mathrm{N}, 78^{\circ} 37^{\prime} \mathrm{W}, 1200 \mathrm{~m}$ (Fig. 2).

Paratypes (48).-ECUADOR (32). Type locality: five $0^{\star \prime}$, six $९$ (EPN 2269, 2270, 2276, 2279-2281, 2283, 2284 2287-2289; collected 23-26

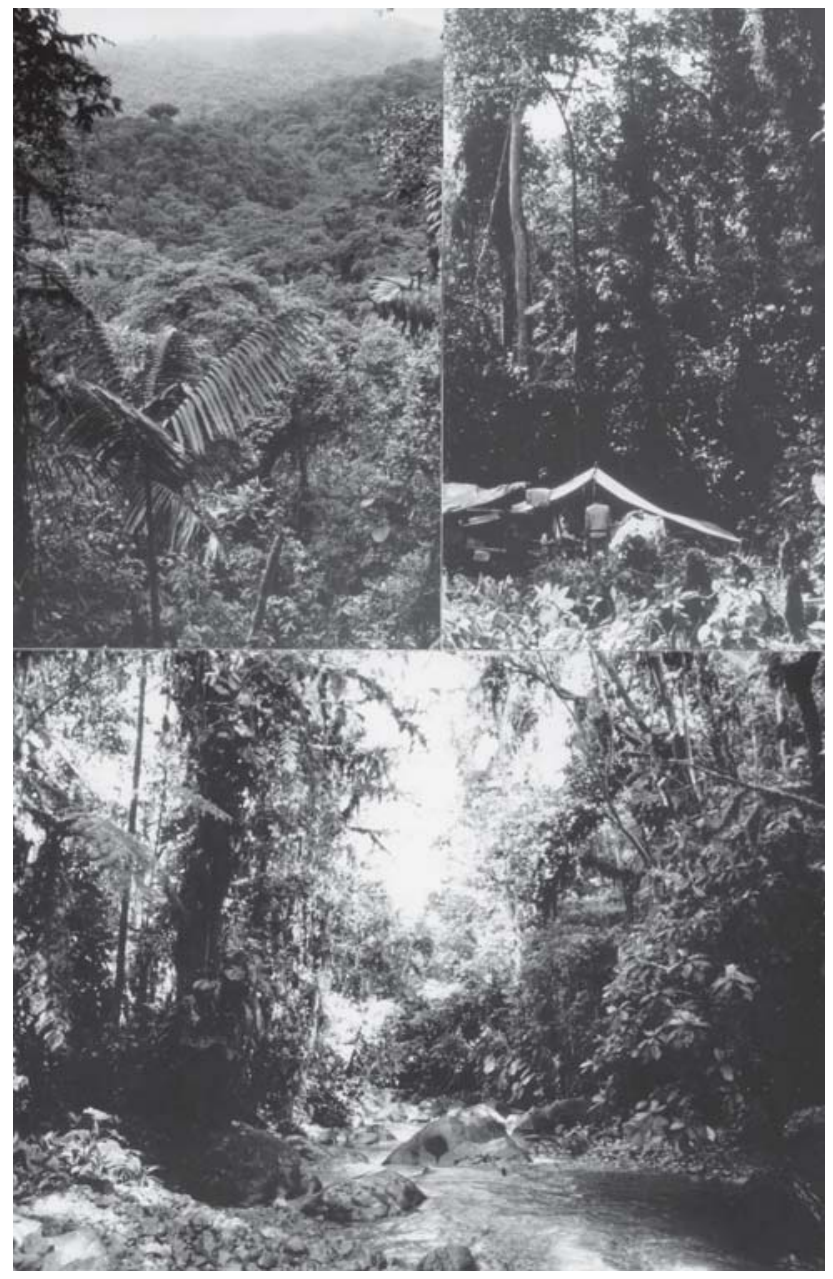

Fig. 2.-Upper left, view of premontane forest cover of Reserva Ecológica Cotacachi-Cayapas in westward direction. Lower view is riparian vegetation of type locality. Upper right is forest profile at Los Pambiles.

July 1985 by P. Mena V. and J. Regalado B.); eight Ơ, 12 ९ (CM 112804-112821, EPN 9771, 9772 collected 23-27 November 1991 by I. Manzano and T.J. McCarthy; Fig. 1, locality 8). Provincia de Chimborazo: Pallatanga, $01^{\circ} 59^{\prime} \mathrm{S}, 78^{\circ} 57^{\prime} \mathrm{W}$, ca. $1200 \mathrm{~m}$; one $ᄋ$ (EPN 67.22; collected 27 February 1967 by M. Olalla; Fig. 1, locality 9). COLOMBIA (16; arranged north to south). Departamento de Chocó: $4 \mathrm{~km}$ N La Italia, $04^{\circ} 56^{\prime} 45^{\prime \prime} \mathrm{N}, 76^{\circ} 25^{\prime} 08^{\prime \prime} \mathrm{W}, 300 \mathrm{~m}$., one ơ (UV 10019; collected 6 September 1988 by M.S. Alberico; Fig. 1, locality 1). Chocó: Alto de Galapagos, near San José del Palmar, 04 $52^{\prime} 47^{\prime \prime N}, 76^{\circ} 11^{\prime} 01^{\prime \prime W}$, $2000 \mathrm{~m}$, three (UV 4440-4442; collected 14 May 1985 by J. Albornoz R. and L.A. Neira; Fig. 1, locality 2). Chocó: Alto de Oso, 8 km W La Italia, $04^{\circ} 52^{\prime} 10^{\prime \prime} \mathrm{N}, 76^{\circ} 11^{\prime} 01^{\prime \prime} \mathrm{W}, 2000 \mathrm{~m}$, one $0^{\prime \prime}$, one (UV 7443, 7445; collected 2 August 1986 by M. S. Alberico; Fig. 1, locality 3). Departamento del Valle del Cauca: Campamento del Río Azul, $03^{\circ} 56^{\prime} 24^{\prime \prime} \mathrm{N}, 76^{\circ} 45^{\prime} \mathrm{W}, 850 \mathrm{~m}$, four Ơ (UV 11556, 11566, 11567, 11569; collected 24, 25 July 1993 by V. Rojas D.; Fig. 1, locality 4). Valle del Cauca: Campamento del Río Azul, confluence of Río Azul at Río Calima, $03^{\circ} 55^{\prime} \mathrm{N}, 76^{\circ} 42^{\prime} \mathrm{W}, 560 \mathrm{~m}$, one O', one $\bigcirc$ (UV 11190, 11198; collected 18 April 1993 by V. Rojas D.; Fig. 1, locality 5). Valle del Cauca: Quebrada de la Delfina, Vereda Aguas Lindas, $3^{\circ} 49^{\prime} 44^{\prime \prime N}, 76^{\circ} 14^{\prime} 11^{\prime \prime W}$, $1200 \mathrm{~m}$, three Ơ (UV 11724-26; collected 23 February 1998 by C.A. Saavedra; Fig. 1, locality 6). Departamento de Nariño: Reserva Natural 


\begin{tabular}{|c|c|c|c|c|c|c|c|c|}
\hline & \multicolumn{2}{|c|}{ Sturnira mordax } & \multicolumn{4}{|c|}{ Sturnira koopmanhilli } & \multicolumn{2}{|c|}{ Sturnira ludovici } \\
\hline & \multirow[b]{2}{*}{$\begin{array}{l}\text { Males } \\
\mathrm{x} \pm \text { S.D. } \\
\text { (Range) } \\
\text { (n) }\end{array}$} & \multirow[b]{2}{*}{$\begin{array}{l}\text { Females } \\
\mathrm{x} \pm \text { S.D. } \\
\text { (Range) } \\
\text { (n) }\end{array}$} & \multicolumn{2}{|c|}{ Ecuador } & \multicolumn{2}{|c|}{ Colombia } & \multirow[b]{2}{*}{$\begin{array}{l}\text { Males } \\
\mathrm{x} \pm \text { S.D. } \\
\text { (Range) } \\
\text { (n) }\end{array}$} & \multirow[b]{2}{*}{$\begin{array}{l}\text { Females } \\
\mathrm{x} \pm \text { S.D. } \\
\text { (Range) } \\
\text { (n) }\end{array}$} \\
\hline & & & $\begin{array}{l}\text { Males } \\
\mathrm{x} \pm \text { S.D. } \\
\text { (Range) } \\
\text { (n) }\end{array}$ & $\begin{array}{l}\text { Females } \\
\mathrm{x} \pm \text { S.D. } \\
\text { (Range) } \\
\text { (n) }\end{array}$ & $\begin{array}{l}\text { Males } \\
\mathrm{x} \pm \text { S.D. } \\
\text { (Range) } \\
\text { (n) }\end{array}$ & $\begin{array}{l}\text { Females } \\
\mathrm{x} \pm \text { S.D. } \\
\text { (Range) } \\
\text { (n) }\end{array}$ & & \\
\hline FA & $\begin{array}{l}47.3 \pm 1.65 \\
(43.0-49.2) \\
(22)\end{array}$ & $\begin{array}{l}46.8 \pm 1.32 \\
(43.6-48.9) \\
(32)\end{array}$ & $\begin{array}{l}51.2 \pm 0.99 \\
(49.2-52.4) \\
(13)\end{array}$ & $\begin{array}{l}50.6 \pm 0.98 \\
(48.1-52.2) \\
(18)\end{array}$ & $\begin{array}{l}51.1 \pm 0.59 \\
(50.0-51.9) \\
(8)\end{array}$ & $\begin{array}{l}50.1 \pm 0.28 \\
(49.8-50.4) \\
(4)\end{array}$ & $\begin{array}{l}46.6 \pm 1.84 \\
(43.9-49.5) \\
(10)\end{array}$ & $\begin{array}{l}46.7 \pm 1.35 \\
(44.7-48.4) \\
(10)\end{array}$ \\
\hline M III & $\begin{array}{l}47.0 \pm 0.98 \\
(45.1-48.6) \\
(10)\end{array}$ & $\begin{array}{l}46.3 \pm 1.18 \\
(44.2-47.9) \\
(15)\end{array}$ & $\begin{array}{l}49.7 \pm 0.64 \\
(48.5-51.0) \\
(12)\end{array}$ & $\begin{array}{l}48.9 \pm 1.12 \\
(46.9-50.9) \\
(18)\end{array}$ & $\begin{array}{l}50.3 \pm 0.69 \\
(49.2-51.2) \\
(5)\end{array}$ & $\begin{array}{l}48.8 \pm 1.44 \\
(47.1-50.2) \\
(5)\end{array}$ & $\begin{array}{l}45.4 \pm 1.49 \\
(43.6-47.2) \\
(8)\end{array}$ & $\begin{array}{l}45.1 \pm 0.80 \\
(44.0-46.4) \\
(10)\end{array}$ \\
\hline M V & $\begin{array}{l}48.2 \pm 1.04 \\
(45.9-50.0) \\
(10)\end{array}$ & $\begin{array}{l}47.6 \pm 1.28 \\
(45.6-49.6) \\
(15)\end{array}$ & $\begin{array}{l}51.7 \pm 0.70 \\
(50.6-52.8) \\
(12)\end{array}$ & $\begin{array}{l}50.5 \pm 1.48 \\
(48.1-53.5) \\
(18)\end{array}$ & $\begin{array}{l}52.7 \pm 0.40 \\
(52.1-53.1) \\
(5)\end{array}$ & $\begin{array}{l}50.4 \pm 1.13 \\
(49.2-51.7) \\
(5)\end{array}$ & $\begin{array}{l}46.8 \pm 1.93 \\
(44.5-49.3) \\
(8)\end{array}$ & $\begin{array}{l}46.4 \pm 1.37 \\
(43.5-48.2) \\
(10)\end{array}$ \\
\hline III Ph1 & $\begin{array}{l}18.7 \pm 0.59 \\
(17.7-20.1) \\
(10)\end{array}$ & $\begin{array}{l}18.3 \pm 0.69 \\
(17.1-19.4) \\
(15)\end{array}$ & $\begin{array}{l}19.1 \pm 0.57 \\
(18.2-20.3) \\
(12)\end{array}$ & $\begin{array}{l}18.9 \pm 0.58 \\
(18.2-20.2) \\
(18)\end{array}$ & $\begin{array}{l}19.2 \pm 0.79 \\
(18.0-20.2) \\
(5)\end{array}$ & $\begin{array}{l}18.5 \pm 0.32 \\
(18.0-18.9) \\
(5)\end{array}$ & $\begin{array}{l}17.4 \pm 0.93 \\
(16.2-18.4) \\
(8)\end{array}$ & $\begin{array}{l}17.2 \pm 0.47 \\
(16.2-18.2) \\
(10)\end{array}$ \\
\hline IV $\mathrm{Ph} 1$ & $\begin{array}{l}15.4 \pm 0.55 \\
(14.7-16.1) \\
(10)\end{array}$ & $\begin{array}{l}14.9 \pm 0.66 \\
(13.5-15.7) \\
(15)\end{array}$ & $\begin{array}{l}15.7 \pm 0.52 \\
(15.1-16.7) \\
(12)\end{array}$ & $\begin{array}{l}15.5 \pm 0.64 \\
(14.6-16.7) \\
(18)\end{array}$ & $\begin{array}{l}16.3 \pm 0.48 \\
(15.9-16.9) \\
(5)\end{array}$ & $\begin{array}{l}14.9 \pm 0.48 \\
(14.1-15.3) \\
(5)\end{array}$ & $\begin{array}{l}14.5 \pm 0.86 \\
(13.3-15.3) \\
(8)\end{array}$ & $\begin{array}{l}14.8 \pm 0.72 \\
(13.3-15.9) \\
(10)\end{array}$ \\
\hline V Ph1 & $\begin{array}{l}11.7 \pm 0.53 \\
(11.1-12.3) \\
(10)\end{array}$ & $\begin{array}{l}11.1 \pm 0.47 \\
(10.5-12.2) \\
(15)\end{array}$ & $\begin{array}{l}12.9 \pm 0.56 \\
(11.9-14.0) \\
(12)\end{array}$ & $\begin{array}{l}12.5 \pm 0.70 \\
(11.5-13.9) \\
(18)\end{array}$ & $\begin{array}{l}12.3 \pm 0.69 \\
(11.8-13.3) \\
(5)\end{array}$ & $\begin{array}{l}11.7 \pm 0.71 \\
(10.9-12.9) \\
(5)\end{array}$ & $\begin{array}{l}10.6 \pm 0.68 \\
(9.8-11.8) \\
(8)\end{array}$ & $\begin{array}{l}11.1 \pm 0.76 \\
(10.1-12.8) \\
(10)\end{array}$ \\
\hline $\mathrm{III} \mathrm{Ph} 2$ & $\begin{array}{l}23.3 \pm 0.86 \\
(21.7-24.4) \\
(10)\end{array}$ & $\begin{array}{l}22.6 \pm 0.73 \\
(21.3-23.7) \\
(13)\end{array}$ & $\begin{array}{l}25.2 \pm 0.68 \\
(24.2-26.5) \\
(12)\end{array}$ & $\begin{array}{l}24.8 \pm 0.70 \\
(23.2-26.2) \\
(18)\end{array}$ & $\begin{array}{l}25.9 \pm 0.34 \\
(25.4-26.4) \\
(5)\end{array}$ & $\begin{array}{l}24.8 \pm 0.45 \\
(24.4-25.4) \\
(5)\end{array}$ & $\begin{array}{l}22.2 \pm 1.19 \\
(20.4-23.3) \\
(8)\end{array}$ & $\begin{array}{l}22.2 \pm 0.75 \\
(20.8-23.4) \\
(10)\end{array}$ \\
\hline TB & $\begin{array}{l}20.7 \pm 0.58 \\
(19.5-21.5) \\
(10)\end{array}$ & $\begin{array}{l}20.5 \pm 0.89 \\
(18.6-21.9) \\
(15)\end{array}$ & $\begin{array}{l}22.5 \pm 0.53 \\
(21.8-23.0) \\
(7)\end{array}$ & $\begin{array}{l}22.1 \pm 0.53 \\
(20.8-22.9) \\
(12)\end{array}$ & $\begin{array}{l}- \\
- \\
-\end{array}$ & $\begin{array}{l}- \\
- \\
-\end{array}$ & $\begin{array}{l}19.5 \pm 0.85 \\
(18.5-20.1) \\
(3)\end{array}$ & $\begin{array}{l}19.0 \pm 0.38 \\
(18.5-19.5) \\
(6)\end{array}$ \\
\hline $\mathrm{TL}$ & $\begin{array}{l}74.5 \pm 3.14 \\
(70.0-80.0) \\
(12)\end{array}$ & $\begin{array}{l}74.1 \pm 3.99 \\
(68.0-82.0) \\
(21)\end{array}$ & $\begin{array}{l}76.8 \pm 1.75 \\
(74.0-78.5) \\
(6)\end{array}$ & $\begin{array}{l}74.5 \pm 2.14 \\
(72.5-77.0) \\
(7)\end{array}$ & $\begin{array}{l}83.5 \pm 3.31 \\
(81.0-88.0) \\
(4)\end{array}$ & $\begin{array}{l}75.3 \pm 3.40 \\
(72.0-80.0) \\
(4)\end{array}$ & $\begin{array}{l}73.3 \pm 2.38 \\
(71.0-76.0) \\
(5)\end{array}$ & $\begin{array}{l}72.4 \pm 2.07 \\
(69.0-74.0) \\
(5)\end{array}$ \\
\hline $\mathrm{HF}$ & $\begin{array}{l}14.6 \pm 1.12 \\
(12.0-16.0) \\
(13)\end{array}$ & $\begin{array}{l}14.0 \pm 1.21 \\
(12.0-16.0) \\
(23)\end{array}$ & $\begin{array}{l}17.1 \pm 0.49 \\
(16.5-18.0) \\
(6)\end{array}$ & $\begin{array}{l}16.5 \pm 0.65 \\
(16.0-17.5) \\
(7)\end{array}$ & $\begin{array}{l}14.3 \pm 2.15 \\
(11.0-17.0) \\
(4)\end{array}$ & $\begin{array}{l}17.5 \pm 1.00 \\
(17.0-19.0) \\
(4)\end{array}$ & $\begin{array}{l}15.6 \pm 0.89 \\
(15.0-17.0) \\
(5)\end{array}$ & $\begin{array}{l}15.7 \pm 0.44 \\
(15.0-16.0) \\
(5)\end{array}$ \\
\hline $\mathrm{E}$ & $\begin{array}{l}17.2 \pm 1.07 \\
(16.0-19.0) \\
(13)\end{array}$ & $\begin{array}{l}16.8 \pm 1.19 \\
(15.0-19.0) \\
(23)\end{array}$ & $\begin{array}{l}19.3 \pm 0.27 \\
(19.0-19.5) \\
(6)\end{array}$ & $\begin{array}{l}19.1 \pm 0.35 \\
(18.5-19.5) \\
(7)\end{array}$ & $\begin{array}{l}17.8 \pm 3.30 \\
(13.0-20.0) \\
(4)\end{array}$ & $\begin{array}{l}19.8 \pm 0.50 \\
(19.0-20.0) \\
(4)\end{array}$ & $\begin{array}{l}17.7 \pm 0.83 \\
(17.0-19.0) \\
(5)\end{array}$ & $\begin{array}{l}17.4 \pm 0.65 \\
(17.0-18.5) \\
(5)\end{array}$ \\
\hline MASS & $\begin{array}{l}26.3 \pm 1.70 \\
(25.0-29.0) \\
(7)\end{array}$ & $\begin{array}{l}26.8 \pm 1.89 \\
(24.0-28.0) \\
(4)\end{array}$ & $\begin{array}{l}33.2 \pm 1.77 \\
(30.0-36.0) \\
(13)\end{array}$ & $\begin{array}{l}28.4 \pm 1.58 \\
(25.5-31.5) \\
(12)\end{array}$ & $\begin{array}{l}- \\
- \\
-\end{array}$ & $\begin{array}{l}- \\
- \\
-\end{array}$ & $\begin{array}{l}27.1 \pm 2.57 \\
(24.0-30.5) \\
(9)\end{array}$ & $\begin{array}{l}(22.0,22.5) \\
(2)\end{array}$ \\
\hline
\end{tabular}




\begin{tabular}{|c|c|c|c|c|c|c|c|c|}
\hline & \multicolumn{2}{|c|}{ Sturnira mordax } & \multicolumn{4}{|c|}{ Sturnira koopmanhilli } & \multicolumn{2}{|c|}{ Sturnira ludovici } \\
\hline & \multirow[b]{2}{*}{$\begin{array}{l}\text { Males } \\
\mathrm{x} \pm \text { S.D. } \\
\text { (Range) } \\
\text { (n) }\end{array}$} & \multirow[b]{2}{*}{$\begin{array}{l}\text { Females } \\
\mathrm{x} \pm \text { S.D. } \\
\text { (Range) } \\
\text { (n) }\end{array}$} & \multicolumn{2}{|c|}{ Ecuador } & \multicolumn{2}{|c|}{ Colombia } & \multirow[b]{2}{*}{$\begin{array}{l}\text { Males } \\
\mathrm{X} \pm \text { S.D. } \\
\text { (Range) } \\
\text { (n) }\end{array}$} & \multirow[b]{2}{*}{$\begin{array}{l}\text { Females } \\
\mathrm{x} \pm \text { S.D. } \\
\text { (Range) } \\
\text { (n) }\end{array}$} \\
\hline & & & $\begin{array}{l}\text { Males } \\
\mathrm{x} \pm \text { S.D. } \\
\text { (Range) } \\
\text { (n) }\end{array}$ & $\begin{array}{l}\text { Females } \\
\mathrm{x} \pm \text { S.D. } \\
\text { (Range) } \\
\text { (n) }\end{array}$ & $\begin{array}{l}\text { Males } \\
\mathrm{x} \pm \text { S.D. } \\
\text { (Range) } \\
\text { (n) }\end{array}$ & $\begin{array}{l}\text { Females } \\
\mathrm{x} \pm \text { S.D. } \\
\text { (Range) } \\
(\mathrm{n})\end{array}$ & & \\
\hline GLS & $\begin{array}{l}24.5 \pm 0.88 \\
(22.8-25.9) \\
(22)\end{array}$ & $\begin{array}{l}23.9 \pm 0.91 \\
(22.3-25.3) \\
(23)\end{array}$ & $\begin{array}{l}25.7 \pm 0.25 \\
(25.1-26.3) \\
(13)\end{array}$ & $\begin{array}{l}25.2 \pm 0.51 \\
(24.5-26.0) \\
(19)\end{array}$ & $\begin{array}{l}25.6 \pm 0.35 \\
(25.0-26.0) \\
(9)\end{array}$ & $\begin{array}{l}25.0 \pm 0.31 \\
(24.7-25.5) \\
(5)\end{array}$ & $\begin{array}{l}24.2 \pm 0.47 \\
(23.4-25.6) \\
(16)\end{array}$ & $\begin{array}{l}23.8 \pm 0.56 \\
(23.1-24.6) \\
(14)\end{array}$ \\
\hline $\mathrm{ZB}$ & $\begin{array}{l}13.2 \pm 0.38 \\
(12.4-13.9) \\
(22)\end{array}$ & $\begin{array}{l}12.8 \pm 0.41 \\
(12.2-13.5) \\
(21)\end{array}$ & $\begin{array}{l}14.6 \pm 0.29 \\
(14.0-15.1) \\
(13)\end{array}$ & $\begin{array}{l}14.1 \pm 0.34 \\
(13.4-14.8) \\
(17)\end{array}$ & $\begin{array}{l}14.9 \pm 0.44 \\
(14.3-15.7) \\
(8)\end{array}$ & $\begin{array}{l}14.0 \pm 0.25 \\
(13.7-14.2) \\
(3)\end{array}$ & $\begin{array}{l}14.2 \pm 0.19 \\
(13.8-14.6) \\
(15)\end{array}$ & $\begin{array}{l}13.8 \pm 0.34 \\
(13.0-14.3) \\
(13)\end{array}$ \\
\hline $\mathrm{BB}$ & $\begin{array}{l}10.7 \pm 0.27 \\
(10.2-11.5) \\
(22)\end{array}$ & $\begin{array}{l}10.5 \pm 0.28 \\
(10.0-10.9) \\
(22)\end{array}$ & $\begin{array}{l}11.5 \pm 0.17 \\
(11.3-11.7) \\
(13)\end{array}$ & $\begin{array}{l}11.2 \pm 0.21 \\
(10.9-11.9) \\
(19)\end{array}$ & $\begin{array}{l}11.6 \pm 0.18 \\
(11.2-11.8) \\
(9)\end{array}$ & $\begin{array}{l}11.2 \pm 0.14 \\
(11.0-11.3) \\
(5)\end{array}$ & $\begin{array}{l}10.8 \pm 0.19 \\
(10.0-11.2) \\
(16)\end{array}$ & $\begin{array}{l}10.7 \pm 0.23 \\
(10.3-11.1) \\
(14)\end{array}$ \\
\hline MB & $\begin{array}{l}12.1 \pm 0.42 \\
(11.5-12.8) \\
(22)\end{array}$ & $\begin{array}{l}11.9 \pm(0.42) \\
(11.1-12.5) \\
(22)\end{array}$ & $\begin{array}{l}13.3 \pm 0.25 \\
(12.7-13.8) \\
(12)\end{array}$ & $\begin{array}{l}12.8 \pm 0.32 \\
(12.1-13.2) \\
(19)\end{array}$ & $\begin{array}{l}13.3 \pm 0.27 \\
(13.0-13.8) \\
(9)\end{array}$ & $\begin{array}{l}13.1 \pm 0.20 \\
(12.8-13.3) \\
(5)\end{array}$ & $\begin{array}{l}12.5 \pm 0.31 \\
(11.9-12.9) \\
(16)\end{array}$ & $\begin{array}{l}12.2 \pm 0.23 \\
(11.7-12.4) \\
(14)\end{array}$ \\
\hline POC & $\begin{array}{l}5.9 \pm 0.16 \\
(5.7-6.2) \\
(22)\end{array}$ & $\begin{array}{l}5.8 \pm 0.13 \\
(5.6-6.1) \\
(23)\end{array}$ & $\begin{array}{l}6.4 \pm 0.14 \\
(6.1-6.6) \\
(13)\end{array}$ & $\begin{array}{l}6.2 \pm 0.14 \\
(5.9-6.5) \\
(19)\end{array}$ & $\begin{array}{l}6.2 \pm 0.16 \\
(6.0-6.5) \\
(9)\end{array}$ & $\begin{array}{l}6.1 \pm 0.21 \\
(5.9-6.4) \\
(5)\end{array}$ & $\begin{array}{l}6.1 \pm 0.21 \\
(5.7-6.5) \\
(16)\end{array}$ & $\begin{array}{l}6.1 \pm 0.30 \\
(5.2-6.5) \\
(14)\end{array}$ \\
\hline $\mathrm{BD}$ & $\begin{array}{l}9.4 \pm 0.36 \\
(8.9-10.1) \\
(22)\end{array}$ & $\begin{array}{l}9.2 \pm 0.48 \\
(8.4-10.0) \\
(22)\end{array}$ & $\begin{array}{l}9.8 \pm 0.28 \\
(9.4-10.2) \\
(13)\end{array}$ & $\begin{array}{l}9.5 \pm 0.19 \\
(9.3-10.0) \\
(19)\end{array}$ & $\begin{array}{l}9.8 \pm 0.4 \\
(9.1-10.3) \\
(9)\end{array}$ & $\begin{array}{l}9.6 \pm 0.17 \\
(9.6-9.7) \\
(4)\end{array}$ & $\begin{array}{l}9.2 \pm 0.32 \\
(8.4-9.9) \\
(16)\end{array}$ & $\begin{array}{l}9.2 \pm 0.25 \\
(8.8-9.8) \\
(14)\end{array}$ \\
\hline PL & $\begin{array}{l}10.1 \pm 0.42 \\
(9.2-10.7) \\
(22)\end{array}$ & $\begin{array}{l}9.8 \pm 0.50 \\
(8.8-10.7) \\
(23)\end{array}$ & $\begin{array}{l}10.8 \pm 0.19 \\
(10.4-11.1) \\
(13)\end{array}$ & $\begin{array}{l}10.5 \pm 0.26 \\
(10.0-10.8) \\
(19)\end{array}$ & $\begin{array}{l}10.7 \pm 0.42 \\
(10.1-11.1) \\
(9)\end{array}$ & $\begin{array}{l}10.8 \pm 0.25 \\
(10.6-11.1) \\
(5)\end{array}$ & $\begin{array}{l}9.7 \pm 0.22 \\
(9.5-9.9) \\
(16)\end{array}$ & $\begin{array}{l}9.4 \pm 0.28 \\
(8.8-9.9) \\
(14)\end{array}$ \\
\hline PPL & $\begin{array}{l}9.0 \pm 0.49 \\
(8.1-9.8) \\
(22)\end{array}$ & $\begin{array}{l}8.8 \pm 0.42 \\
(8.1-9.5) \\
(22)\end{array}$ & $\begin{array}{l}9.4 \pm 0.23 \\
(9.1-9.9) \\
(13)\end{array}$ & $\begin{array}{l}9.2 \pm 0.29 \\
(8.8-9.8) \\
(19)\end{array}$ & $\begin{array}{l}9.2 \pm 0.26 \\
(9.0-9.9) \\
(9)\end{array}$ & $\begin{array}{l}8.7 \pm 0.16 \\
(8.5-8.9) \\
(5)\end{array}$ & $\begin{array}{l}9.4 \pm 0.30 \\
(9.0-10.2) \\
(15)\end{array}$ & $\begin{array}{l}9.3 \pm 0.27 \\
(8.8-9.7) \\
(12)\end{array}$ \\
\hline CM3 & $\begin{array}{l}6.9 \pm 0.35 \\
(6.4-7.9) \\
(22)\end{array}$ & $\begin{array}{l}6.7 \pm 0.29 \\
(6.3-7.1) \\
(23)\end{array}$ & $\begin{array}{l}7.3 \pm 0.10 \\
(7.1-7.5) \\
(12)\end{array}$ & $\begin{array}{l}7.2 \pm 0.14 \\
(7.0-7.5) \\
(15)\end{array}$ & $\begin{array}{l}7.1 \pm 0.14 \\
(6.9-7.3) \\
(9)\end{array}$ & $\begin{array}{l}7.1 \pm 0.13 \\
(7.0-7.3) \\
(5)\end{array}$ & $\begin{array}{l}6.8 \pm 0.18 \\
(6.6-7.1) \\
(16)\end{array}$ & $\begin{array}{l}6.8 \pm 0.12 \\
(6.6-7.0) \\
(14)\end{array}$ \\
\hline MM & $\begin{array}{l}7.9 \pm 0.22 \\
(7.5-8.3) \\
(22)\end{array}$ & $\begin{array}{l}7.7 \pm 0.26 \\
(7.2-8.0) \\
(23)\end{array}$ & $\begin{array}{l}8.1 \pm 0.12 \\
(7.9-8.3) \\
(13)\end{array}$ & $\begin{array}{l}8.0 \pm 0.17 \\
(7.8-8.2) \\
(15)\end{array}$ & $\begin{array}{l}8.1 \pm 0.23 \\
(7.7-8.4) \\
(9)\end{array}$ & $\begin{array}{l}7.5 \pm 0.25 \\
(7.2-7.7) \\
(5)\end{array}$ & $\begin{array}{l}8.3 \pm 0.14 \\
(8.0-8.5) \\
(16)\end{array}$ & $\begin{array}{l}8.2 \pm 0.17 \\
(7.9-8.5) \\
(14)\end{array}$ \\
\hline $\mathrm{CC}$ & $\begin{array}{l}6.2 \pm 0.25 \\
(5.8-6.6) \\
(22)\end{array}$ & $\begin{array}{l}5.9 \pm 0.25 \\
(5.5-6.5) \\
(22)\end{array}$ & $\begin{array}{l}6.5 \pm 0.16 \\
(6.3-6.9) \\
(13)\end{array}$ & $\begin{array}{l}6.3 \pm 0.16 \\
(6.1-6.7) \\
(15)\end{array}$ & $\begin{array}{l}6.7 \pm 0.24 \\
(6.4-7.0) \\
(9)\end{array}$ & $\begin{array}{l}6.5 \pm 0.17 \\
(6.3-6.7) \\
(4)\end{array}$ & $\begin{array}{l}6.6 \pm 0.15 \\
(6.2-6.8) \\
(14)\end{array}$ & $\begin{array}{l}6.6 \pm 0.13 \\
(6.0-6.4) \\
(14)\end{array}$ \\
\hline $\mathrm{CL}$ & $\begin{array}{l}3.1 \pm 0.12 \\
(2.8-3.3) \\
(22)\end{array}$ & $\begin{array}{l}2.6 \pm 0.16 \\
(2.2-2.9) \\
(23)\end{array}$ & $\begin{array}{l}3.4 \pm 0.08 \\
(3.3-3.5) \\
(13)\end{array}$ & $\begin{array}{l}2.8 \pm 0.14 \\
(2.6-3.1) \\
(19)\end{array}$ & $\begin{array}{l}3.5 \pm 0.11 \\
(3.3-3.6) \\
(9)\end{array}$ & $\begin{array}{l}2.9 \pm 0.14 \\
(2.8-3.1) \\
(5)\end{array}$ & $\begin{array}{l}3.2 \pm 0.12 \\
(3.0-3.4) \\
(16)\end{array}$ & $\begin{array}{l}2.6 \pm 0.08 \\
(2.4-2.8) \\
(14)\end{array}$ \\
\hline ML & $\begin{array}{l}16.1 \pm 0.56 \\
(15.4-17.0) \\
(22)\end{array}$ & $\begin{array}{l}15.7 \pm 0.60 \\
(14.8-16.9) \\
(23)\end{array}$ & $\begin{array}{l}17.0 \pm 0.22 \\
(16.5-17.4) \\
(13)\end{array}$ & $\begin{array}{l}16.5 \pm 0.37 \\
(15.5-17.0) \\
(19)\end{array}$ & $\begin{array}{l}17.1 \pm 0.32 \\
(16.8-17.6) \\
(9)\end{array}$ & $\begin{array}{l}16.7 \pm 0.18 \\
(16.4-16.9) \\
(5)\end{array}$ & $\begin{array}{l}15.8 \pm 0.39 \\
(15.3-16.8) \\
(16)\end{array}$ & $\begin{array}{l}15.5 \pm 0.25 \\
(15.1-15.9) \\
(13)\end{array}$ \\
\hline $\mathrm{CPH}$ & $\begin{array}{l}5.5 \pm 0.26 \\
(4.9-5.9) \\
(22)\end{array}$ & $\begin{array}{l}5.3 \pm 0.26 \\
(4.9-5.8) \\
(23)\end{array}$ & $\begin{array}{l}6.2 \pm 0.22 \\
(5.9-6.6) \\
(13)\end{array}$ & $\begin{array}{l}6.0 \pm 0.26 \\
(5.6-6.8) \\
(19)\end{array}$ & $\begin{array}{l}6.1 \pm 0.15 \\
(5.9-6.3) \\
(9)\end{array}$ & $\begin{array}{l}5.7 \pm 0.09 \\
(5.6-5.8) \\
(5)\end{array}$ & $\begin{array}{l}5.6 \pm 0.19 \\
(5.2-5.9) \\
(16)\end{array}$ & $\begin{array}{l}5.6 \pm 0.16 \\
(5.2-5.8) \\
(14)\end{array}$ \\
\hline
\end{tabular}




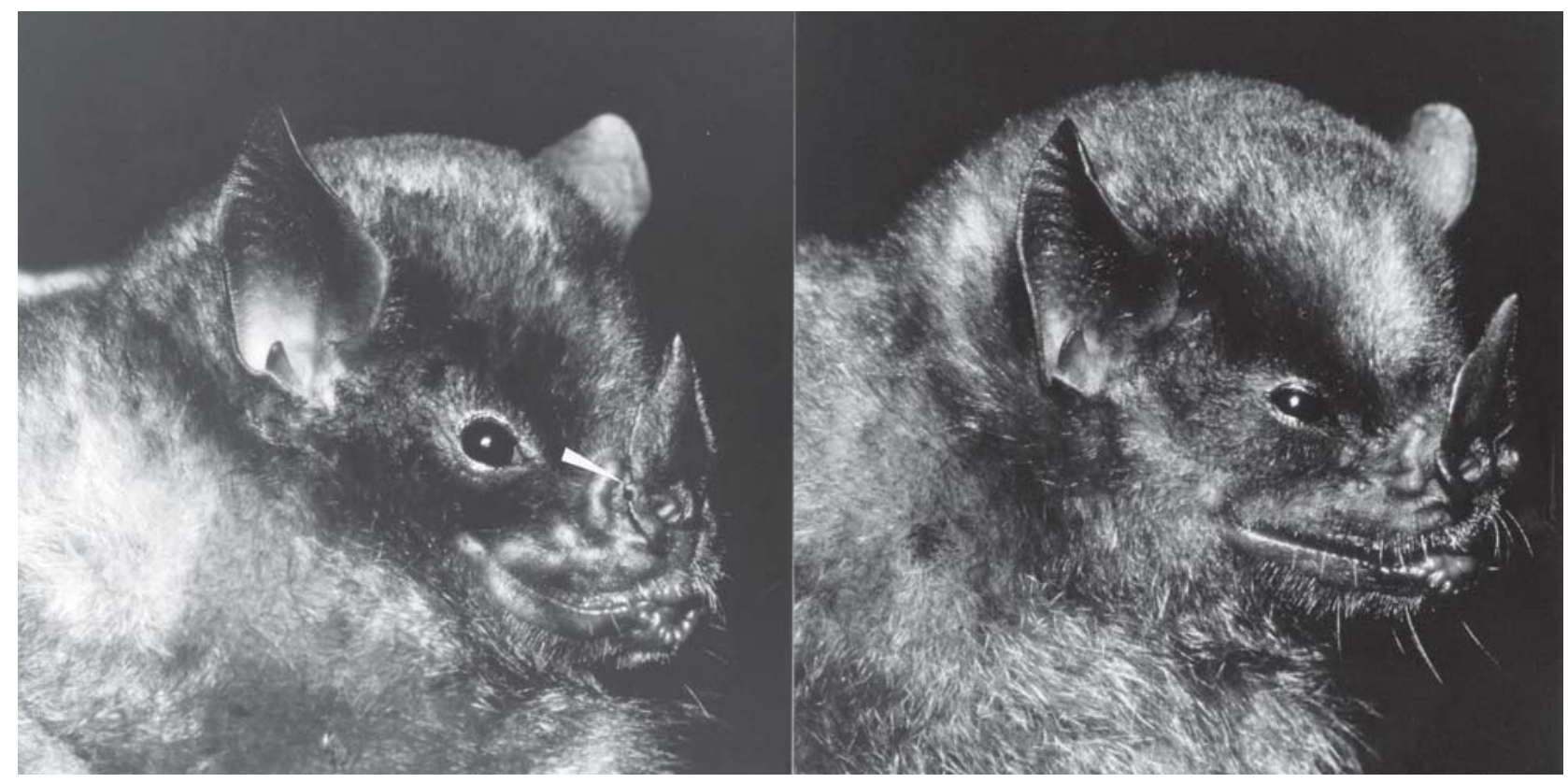

Fig. 3. - Oblique frontal photos of S. koopmanhilli (left) and S. ludovici (right), both sympatric at Los Pambiles. Note contrasting paler dorsal fur and a small, rounded outgrowth (tip of pointer) at the base of the spear on top of the narial pad on each side of the noseleaf structure.

"La Planada," 107'N, 7752'48" W, 1950 m, one Ơ (UV 2926; collected 29 July 1981 by M.S. Alberico; Fig. 1, locality 7).

Distribution.-Pacific lowlands and adjacent slopes of the Andes from the Departamento de Chocó, Colombia, south to Provincia de Chimborazo, Ecuador, ranging from 300 to 2000 m (Fig. 1).

Measurements of Holotype.-FA, 51.6; MIII, 49.5; MV, 51.9; IIIPh1, 19.4; IVPh1, 15.6; VPh1, 12.5, IIIPh2, 25.3; TL, 78.5; HF, 16.5; E, 19.5; MASS, $35.0 \mathrm{~g}$; GLS, 26.3; ZB, 15.0; BB, 11.7; MB, 13.8; POC, 6.4; BD, 10.2; PL, 11.1; PPL, 9.9; CM3, 7.5; MM, 8.1; CC, 6.6; CL, 3.4; ML, $17.4 ; \mathrm{CPH}, 6.1$.

Etymology.-The patronym koopmanhilli honors, posthumously, Karl F. Koopman (American Museum of Natural History) and John Edwards Hill (Natural History Museum, London) for their contributions to chiropteran systematics and their unselfish attention given to colleagues and professional affiliations.

Diagnosis.-A moderately large species of Sturnira (FA 48.1-52.4). Active shoulder glands are present. A small, rounded dermal outgrowth is situated on top of each narial pad along the base of the spear of the noseleaf structure. Dorsal fur appears bicolored. The hind feet are sparsely haired. Cranium is elongated and slender with complete zygomatic arches. The posterior border of the hard palate forms a rounded or disrupted round shape. The recessed hypoglossal foramina cannot be viewed from above the occipital condyles. Upper and lower inner incisors are enlarged and protrudent. A sulcus is present on posterior face of each upper canine. P1 small; P2 larger than M2. Variable spacing is present between premolars and molars in upper and lower tooth rows. Lingual edges of $\mathrm{m} 1$ and $\mathrm{m} 2$ not cuspidate, but appear as low, unbroken ridges.

Description. Measurements.-External and cranial measurements (Tables 1,2) exemplify the size of $S$. koopmanhilli (Ecuador and Colombia combined) across forearm (FA 48.1-52.4), wing (MV 48.1-53.5; IIIPh2 23.2-26.5), and leg (ТВ 20.8-23.0) bones; lengths of cranium (GLS 24.5-26.3) and mandible (ML 15.5-17.6). Mass averages are $33.2 \mathrm{~g}$ (males) and $28.4 \mathrm{~g}$ (females) for nonparous and fasted adults.

Fur- - Color pattern of dorsal hair involves three bands. The epibasal band expresses pale grayish to brownish color not contrasting strongly with the equal or longer subdistal band of white. Proportion and contrast of this reflects a mottled, bicolored appearance on the upper dorsum (Fig. 3). The effect is a predominant light-colored fur with brownish distal tips washed in blackish, reddish, yellowish, or grayish tones. Dorsal (interscapular) fur is lax; hair length ca. $9.0-10.0 \mathrm{~mm}$. The length of the white subdistal band is reduced on the rump hairs; epibasal band darker; rump hairs shorter $(8.5-9.0 \mathrm{~mm})$. Ventral fur is shorter (ca. $5.0 \mathrm{~mm})$; subdistal band darker.

Early gland activity in the shoulder region occurs in both sexes of S. koopmanhilli with hairs tinted light yellow (e.g., CM 112812, 112815). The development of reddish brown to blackish epaulettes occurs in males (e.g., EPN 9770).

The dorsum and ventrum of the lower half of the forearms are lightly furred continuing onto the wing membranes, especially the propatagia. The length of each hind leg and its associated patagia are lightly furred dorsally and moderately below. The rudimentary interfemoral membrane is moderately fringed with short hairs and along lower legs. The edge of each plagiopatagium is lightly fringed; feet are sparsely haired. The wing membranes are black.

Soft anatomy.-The spear of the noseleaf structure is cordate with an acute apex. The horseshoe associated with the noseleaf is reduced to narial pads at each side of the nostril region in Sturnira. A small, rounded dermal outgrowth is situated at each junction of the spear where it joins the narial pad (Fig. 3).

Sturnira koopmanhilli possesses five pairs of soft palatal, crescentshaped ridges. These approach but do not join along the mid-palate. A sixth continuous shelf extends the width of the rear palate. The lateral ends of the five ridge pairs orient along the lingual side of the tooth rows: P2 (1), M1 


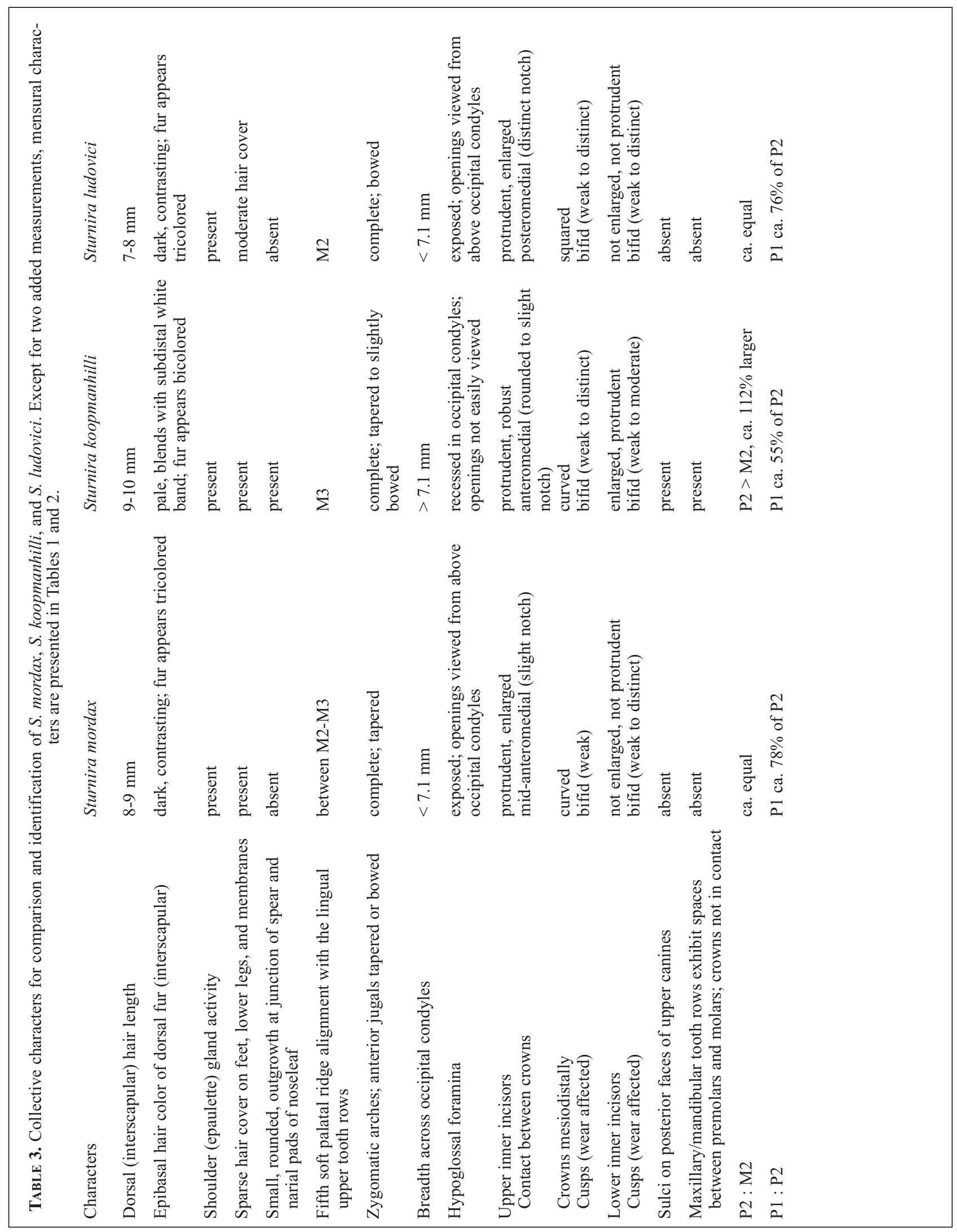




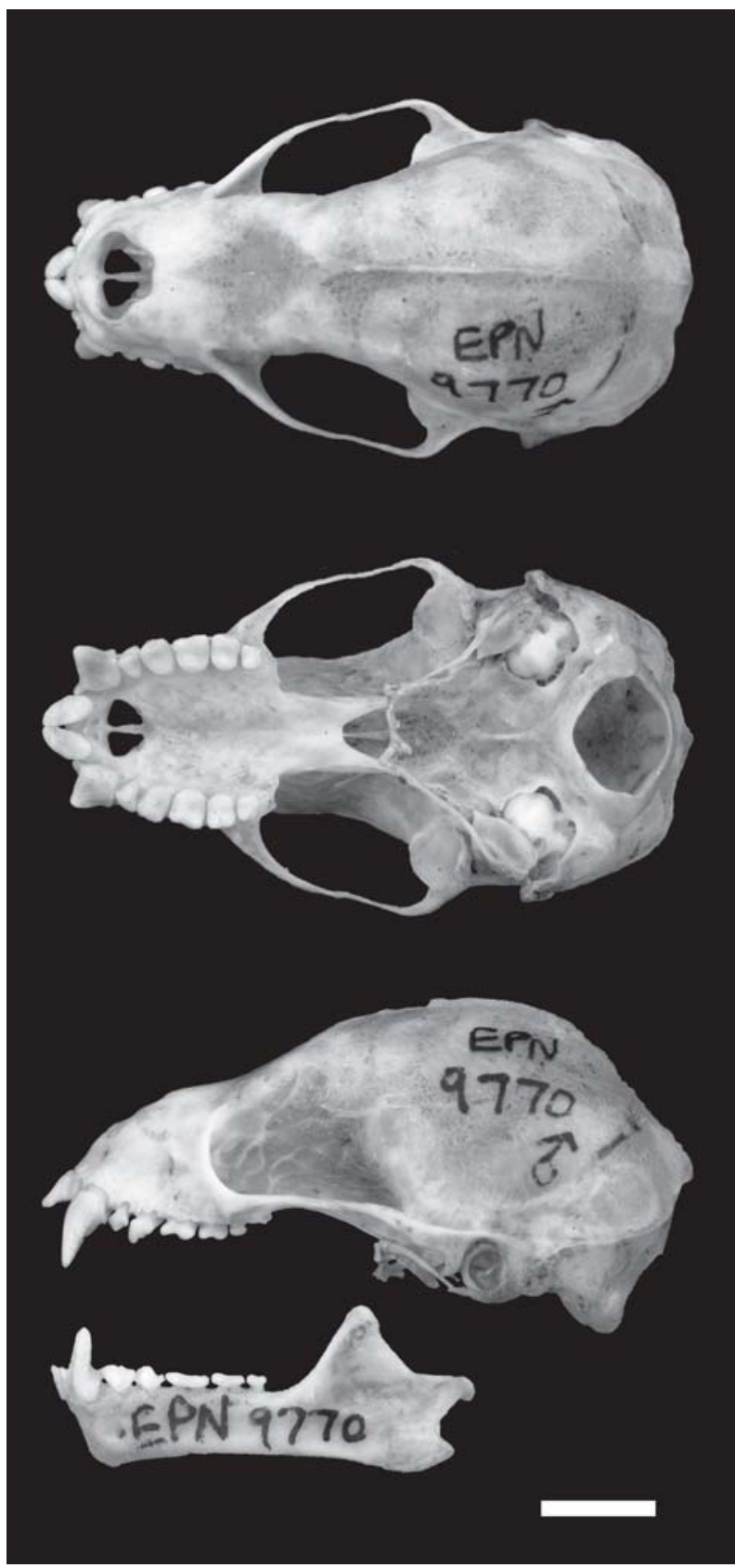

Fig. 4.-Dorsal, ventral, and lateral cranium and lateral mandible profiles of the holotype (EPN 9970) of S. koopmanhilli. Bar equals $5 \mathrm{~mm}$.

(2), M1-2 (3), M2 (4), and M3 (5). The shelf (6) aligns posterior of M3. Skull.-The cranium of S. koopmanhilli is elongated and rather slender (Fig. 4). A longer rostrum is reflected by the mean measurements (Table 2) for PL versus PPL (male, 10.8:9.4; female, 10.5:9.2). The lateral profile of the cranium in $S$. koopmanhilli usually slopes moderately for a relatively large skull (Fig. 4). Zygomatic arches are complete and slender; anterior jugals tapered to slightly bowed. Select measurements of width (means: POC, ZB, BB, MB; Table 2) expressed as percentages of respective greatest length mean (GLS; Table 2) provide a comparison as to indicate shape in S. koopmanhilli (male: $24.9 \%, 56.8 \%, 44.7 \%, 51.7 \%$; female: $24.6 \%, 55.9 \%, 44.4 \%, 50.8 \%$; see COMPARISONS).
The shape of the paired incisive (palatal) foramina in S. koopmanhilli is generally irregular triangles with longer sides extending posteriorly. Nasal septum is slender. The vomerine spine terminates in S. koopman-hilli at the edge of the extended palate or slightly beyond; a rounded or disrupted-round border results, but not $V$-shaped.

The occipital region in $S$. koopmanhilli is enlarged as expressed by depth of braincase (BD, Table 2; Fig. 4); breadth across occipital condyles averages $7.4(\mathrm{n}=12)$. The hypoglossal foramina are recessed at the anterior base of each occipital condyle, which obscure these openings when viewed from above.

Length of mandible (ML, Table 2, Fig. 4) averages 17.0 (male) and 16.5 (female) in $S$. koopmanhilli, while the $\mathrm{CPH}$ averages 6.2 (male) and 6.0 (female). The $\mathrm{CPH}$ as a percentage of $\mathrm{ML}$ is about equal in males $(36.5 \%)$ and females (36.4\%). The coronoid and condylar processes are relatively uniform and robust. The condyle and condylar neck regions are thickened and defined. The posterior slope of the coronoid process is abrupt, usually joining the neck anterior to the condyle without ambiguity. Two of 27 mandibles possessed posterior slopes that were more gradual with ambiguous attachment on the condylar process.

Incisors and canines.-Both I1 and il in S. koopmanhilli are enlarged and protrudent, forming curved incisive outlines that occlude beyond a plane across the anterior faces of the i2 (Figs. 4, 9). The distal coronal cusp is small compared to the enlarged mesial cusp in both upper and lower incisors. Both pairs of incisors contact anteromedially near the incisor tip. Generally the crowns form a rounded tip with a slight medial notch. Elongate, semi-uniform, anterior faces of I1 form a "V" pattern (Fig. 4, 9). The lateral edges of both I1 curve posteriorly. Upper and lower inner incisors express weak to distinct bifid appearances, changing with wear. Incisive cingula are expanded moderately to the base of upper inner incisors. The position and size of the robust I1 in S. koopmanhilli reduces the space between each I1 and canine, tending to crowd the smaller I 2 to the outside of the tooth row. The premaxillary and the mandibular symphysis associated with these incisors are clearly developed. Both I1 and i1 have lingual fossae with marginal ridges encircling the lingual surfaces.

The protruding upper and lower incisors in S. koopmanhilli lend to skull elongation; proportion of the GLS with upper incisors included compared to GLS without incisors add 4.6\% (female) and 4.0\% (male) to length.

A distinctive sulcus is present on each posterior face of the upper canines in S. koopmanhilli. CL average longer in males (Table 2).

Premolars and molars.-Maxillary and mandibular tooth rows in S. koopmanhilli exhibit gaps or diastemata between the premolars and molars (Figs. 4, 5). Slight to distinct spacing between the crowns of P1-M3 and p1-m3 are consistent; single closed spaces in three of 28 specimens. Separation between C-P1 and c-p1 is not stable (14 of 28 specimens).

First upper premolar is small in S. koopmanhilli with a wide, but narrow, protocone region and a low, ridge-like paracone; P1 ca. 54\% the size of P2. Second upper premolar large, approaching $81 \%$ of M1 and ca. $112 \%$ the occlusal size of M2. The P2 with an enlarged protocone contributes to a wide lingual shelf, increasing the molar surface area. Buccal and distostyle cingula are enlarged on P2; enlarged distostylids are present on $\mathrm{p} 1$ and $\mathrm{p} 2$.

Molar topography is low and subtle in S. koopmanhilli. Relative size proportions between the upper molars shows M2 ca. $72 \%$ of M1 and M3 ca. 35\% of M2. When lingual upper molars (Fig. 5) are viewed in profile, these display a large rounded metacone on M1; the paracone is smaller and angular, not rounded. The paracones and metacones of M2 approach similar height and size; the paracone larger and appears rounded; small M3 is ovoid in shape.

Lower molars in S. koopmanhilli are poorly defined, especially in the lingual postion of the metaconid and entoconid. This position on $\mathrm{m} 1$ and $\mathrm{m} 2$ is occupied by a low, ridge sloping between slight indications of cusp development (Fig. 5). Relative size proportion of the $\mathrm{m} 2$ is ca. $69 \%$ of $\mathrm{m} 1$ and the $\mathrm{m} 3$ is ca. $43 \%$ of $\mathrm{m} 2$. The lower molars have buccal and lingual cingula that form low ridges encircling shallow basins or fossae. 

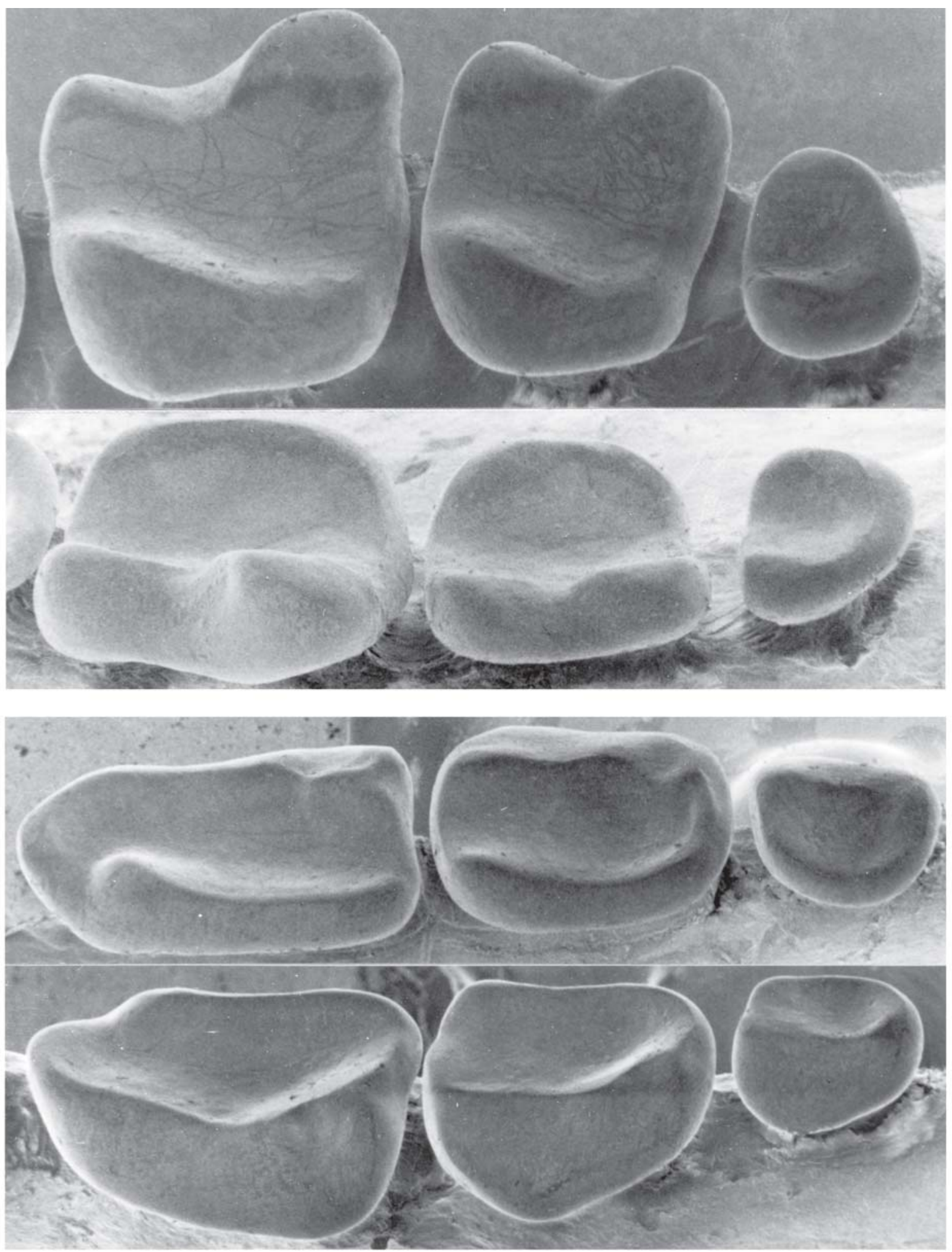

Fig. 5.-Scanning electron photos for S. koopmanhilli (EPN 2276) of the maxillary molars (top photo, lingual view of upper left tooth row; second photo, occlusal view of upper right tooth row). Mandibular molars (third photo, lingual occlusal view of lower right tooth row; bottom photo, buccal angle view of lower left tooth row). Length of mesial edge (m1) to distal edge (m2) measures $3.2 \mathrm{~mm}$. 


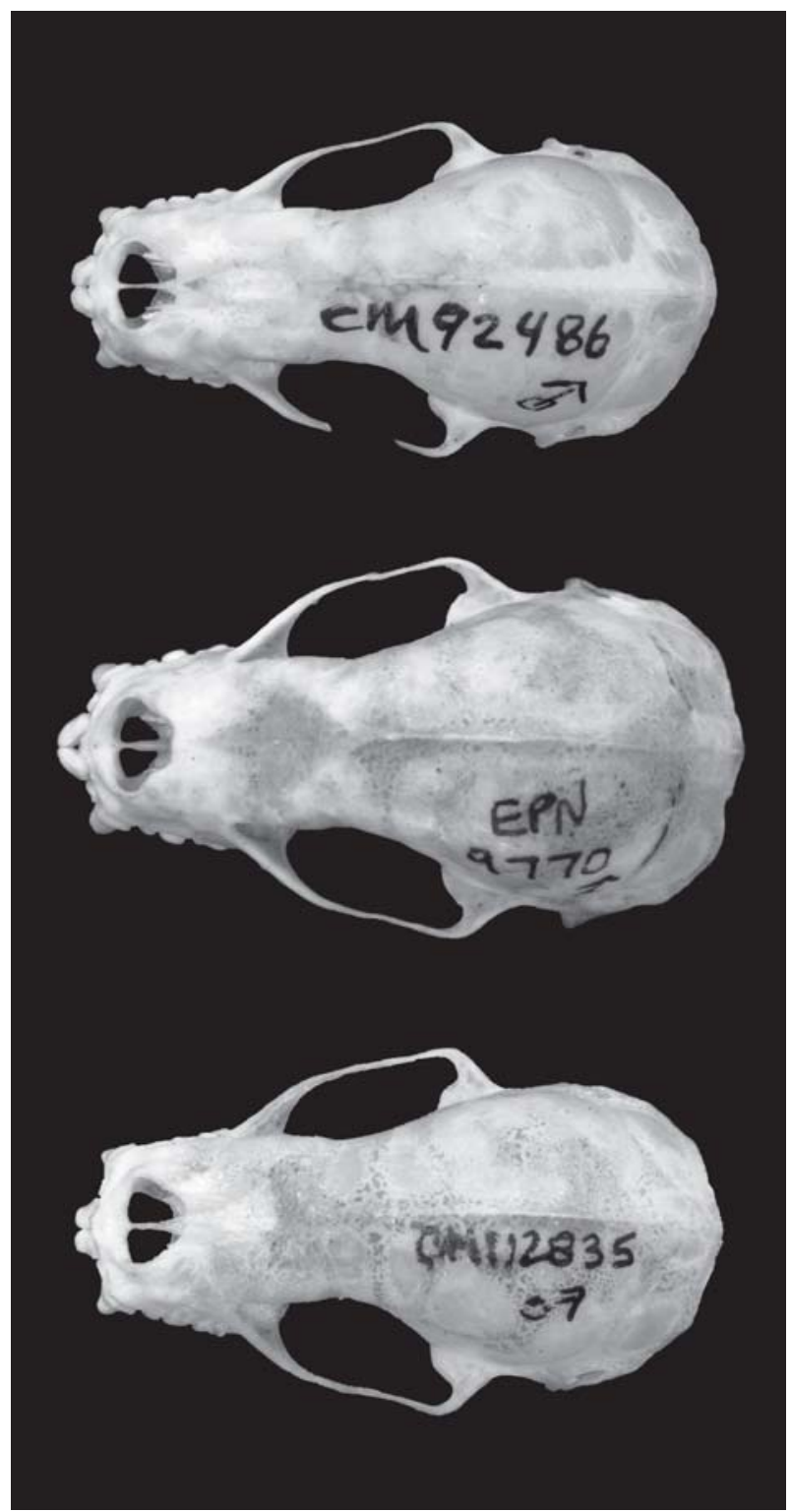

Fig. 6.-Dorsal cranium profiles of S. mordax (CM 92486), S. koopmanhilli (EPN 9970), and S. ludovici (CM 112835).

\section{COMPARISONS}

Sturnira koopmanhilli averages larger than S. mordax and S. ludovici (Tables 1, 2; e.g., FA, MIV, MASS, GLS, ML). Cranial measurements (Table 2; Fig. 6-8) average larger or are essentially equal for PPL (male, female), MM (male, female), and CC (male, female) with S. ludovici. These highlight $S$. koopmanhilli as a moderately large Sturnira. The crania of this bat and S. mordax are similarly more elongated and slender compared with $S$. ludovici (Figs. 6-8). The means for PL:PPL (Table 2) indicate the cranium as less elongated in S. ludovici (male, 9.7:9.4; female, 9.4:9.3) as compared to $S$. mordax (male, 10.1:9.0;

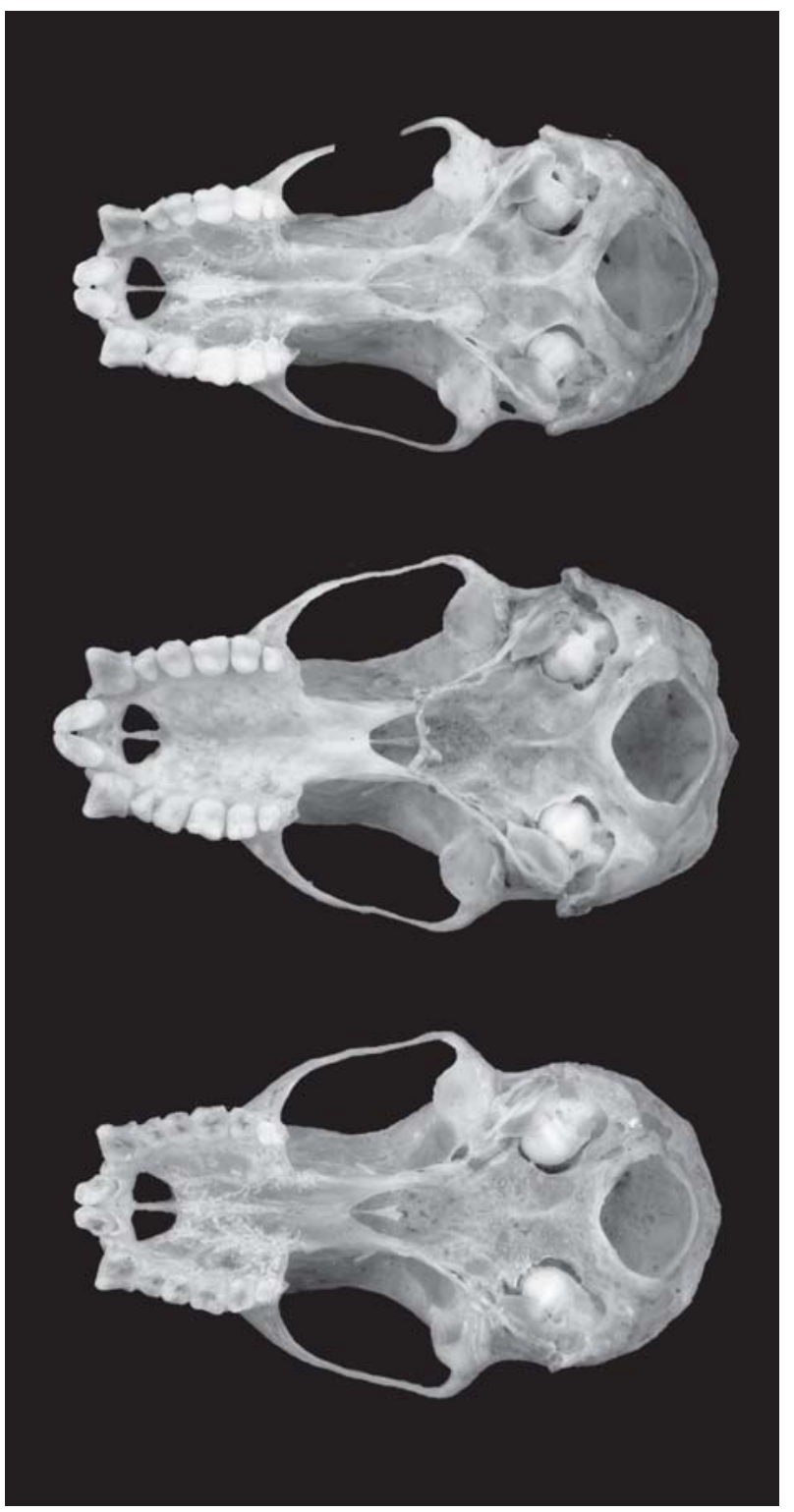

Fig. 7.-Ventral cranium profiles of S. mordax (CM 92486), S. koopmanhilli (EPN 9970), and S. ludovici (CM 112835).

female, 9.8:8.8) and S. koopmanhilli (male, 10.8:9.4; female, 10.5:9.2). Select width measurements (means: POC, $\mathrm{ZB}, \mathrm{BB}, \mathrm{MB}$; Table 2) expressed as percentages of respective GLS (Table 2) support a more slender skull in $S$. mordax (male: $24.1 \%, 53.9 \%, 43.7 \%$, 49.4\%; female: $24.3 \%$, $53.6 \%, 43.9 \%, 49.8 \%$ ) over S. koopmanhilli (see DESCRIPTION) and S. ludovici (male: $25.2 \%, 58.7 \%$, $44.6 \%, 51.7 \%$; female: $25.6 \%, 57.9 \%, 45.0 \%, 51.3 \%$ ).

Characters for the external, skull, and dental anatomy are compared (Table 3) among these three species of Sturnira.

A phylogenetic study of the genus Sturnira (Pacheco and Patterson 1991) included data for one specimen (EPN 67.22), which was reported as an undescribed species 


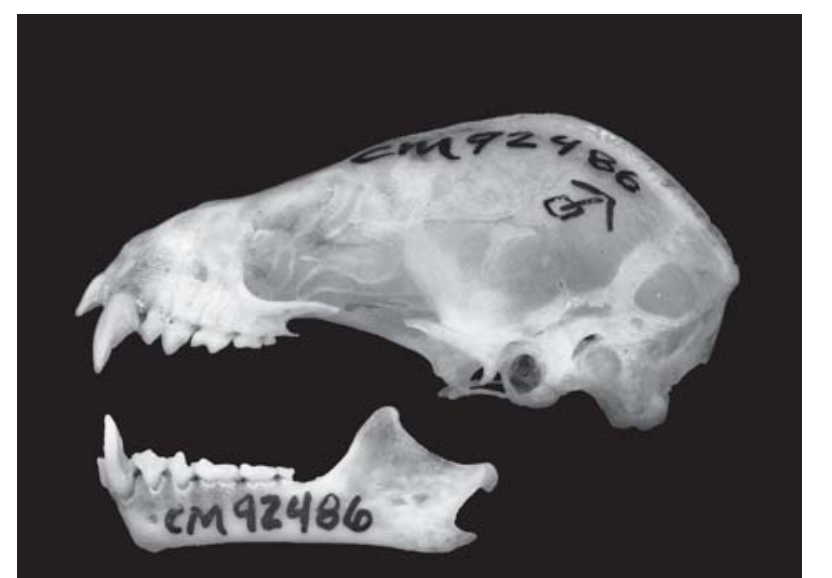

absence of active shoulder glands and incomplete or partial zygomatic arches, which were incorrect. Additional specimens of $S$. koopmanhilli support the presence of median lingual cusp on i1. The occurrence of a posterior internal basal cusp on I1 was reported as absent. This is questionable because the lingual face or fossa of the upper inner incisors is encircled with a continuous rim. The basal cusp may be part of this cingular buildup. These two cusps are ambiguous, certainly not as obvious as those of Corvira. The character of tooth gaps, spaces between premolars and molars in both tooth rows, is present in both S. koopmanhilli and Corvira. Other characters for S. koopmanhilli but not shared with Corvira are the presence of four lower incisors and both I1 and i1 are enlarged and protrudent. Sturnira koopmanhilli is placed in the subgenus Sturnira.

As characterized for S. mordax, Pacheco and Patterson (1991) recorded trifid lower cusps on the lower inner incisors of S. koopmanhilli. McCarthy et al. (2005) felt that wear and age may not explain why examples of adult $S$. mordax possessed a small third cusp on both lower incisors or only one. They suggested that a small third cusp may represent a polymorphic character. We have observed a small additional cusp in instances for $S$. koopmanhilli, but our purpose here is the description of a new taxon. The presence of a third cusp is overshadowed by the bicuspid nature as stable for the lower inner incisors in S. koopmanhilli. This is reflected in Table 3.

Contreras-Vega and Cadena (2000) described S. mistratensis, demonstrating an additional Sturnira with modified and enlarged i1 that protrude. Pacheco and Patterson (1991) recorded this character for Sturnira sp. A. Similar to $S$. mistratensis, the crowns for il are in contact anteromedially and the mesial portions of the crowns are enhanced and protrude in tandem, forming a rounded outline. The lingual cingulum for both species is prolonged to form a tapering body of the incisor.

The presence or absence of distinct to moderate lingual cusps (metaconid and entoconid) on $\mathrm{m} 1$ and $\mathrm{m} 2$ divides the subgenus Sturnira into two groups (Davis 1980; Pacheco and Patterson 1991; Koopman 1994; ContrerasVega and Cadena 2000; Sánchez-Hernández et al. 2005). The grouping of S. lilium, $S$. tildae, S. thomasi, S. aratathomasi, S. luisi, and S. mistratensis comprise the species that share cuspidate lower molars. Those Sturnira that lack cuspidate definition possess a low continuous, sloping ridge on the lingual side. These include $S$. erythromos, $S$. oporaphilum, S. ludovici, S. mordax, S. magna, S. sorianoi, and S. koopmanhilli. We follow Koopman (1994) for the placement of $S$. bogotensis as a subspecies of $S$. oporaphilum. Size as represented by forearm length partitions the remaining congeners. Sexes grouped for $S$. koopmanhilli average 50.5 (females) and 51.1 (males); a combined pool averages 50.8. Our new species is larger than $S$. erythromos (FA 38.0-46.0) S. oporaphilum (44.0-48.0), $S$. sorianoi (41.0-44.0), and as seen for $S$. mordax and $S$. that supported Sturnira sp. A as interm between the two subgenera, Sturnira and Corvira. Certain characters reportedly shared with Corvira included the 

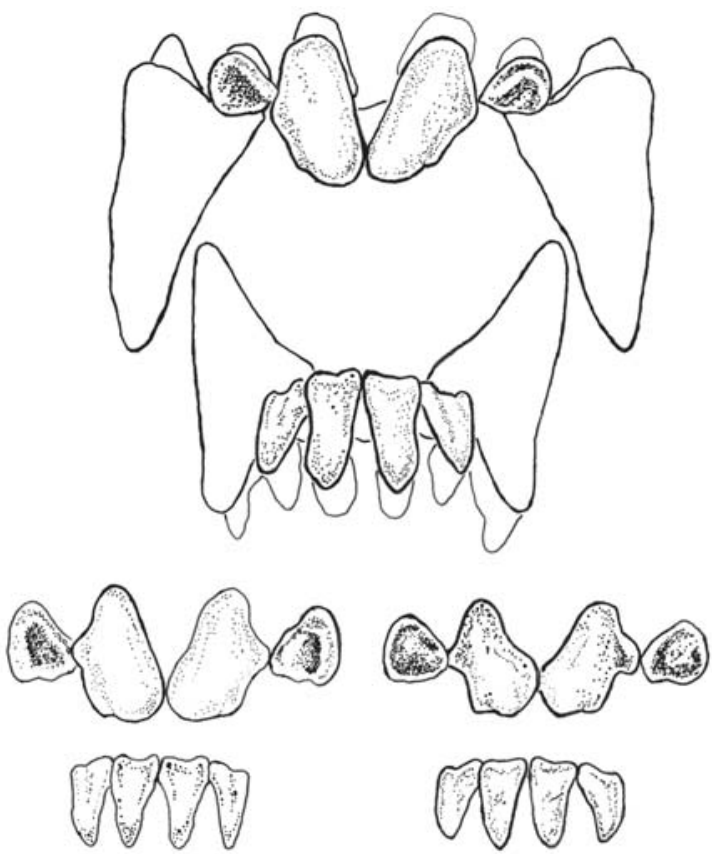

Fig. 9.-Incisors are highlighted for S. koopmanhilli (above, CM 112814), S. mordax (below, left; CM 92486), and S. ludovici (below, right; CM 112835).

ludovici (Table 1; Koopman 1994; Sánchez-Hernández et al. 2005). Similarly, S. magna is larger than S. koopmanhilli (FA > 53.0; Tamsitt and Häuser 1985). Overall, $S$. koopmanhilli is easily distinguishable from all Sturnira when both upper and lower inner incisors are compared. The two sets of incisors are robust, protrudent, and occlude anterior to the incisive tooth row. This combination is unique within Sturnira. The upper and lower premolars and molars are spaced apart so the crowns are not in contact. This character is only known for S. koopmanhilli in the subgenus Sturnira and shared with the subgenus Corvira.

\section{COMMENTS}

Los Pambiles is situated along an irregular ridge of the Cordillera de Toisán. Visits in July 1985 and November 1991 followed a route from Seis de Julio de Cuellaje, Imbabura, $1840 \mathrm{~m}$, ascending the eastern face of the Toisán, then a northwestward descent extending from near $3100 \mathrm{~m}$ to Los Pambiles at $1200 \mathrm{~m}$. The transitional lower montane-premontane forest at Los Pambiles was dominated by trees of medium height $(<25 \mathrm{~m})$, exhibiting two strata that were not always distinct and continuous (Madden and Albuja 1989; Fig. 2), in part, because of the rough topography. Rainfall data are few for the site, where annual precipitation and environmental humidity are high. Madden and Albuja (1989) estimated the annual rainfall as about $4000 \mathrm{~m}$, suggesting that it could be higher on the western Andean slopes of the Toisán. We experienced fog interception, which bathed the site in mist. Albuja and Gardner (2005) reported tree ferns and mosses in the understory and considerable epiphytic growth. See Madden and Albuja (1989) for maps and other information.

Reproductive data for $S$. koopmanhilli remain scant. Two females from late July at Los Pambiles had developing teats and were pregnant with small fetuses, of which one measured $3.0 \mathrm{~mm}$ (crown-rump length). A third female was lactating. In late November, two females were lactating and a third was post lactating. A seemingly adult male (FA 52.0) had incompletely fused manual epiphyses in late November. In Colombia, two females were recorded from Chocó in mid May with a single fetus each $(5.0,20.0 \mathrm{~mm})$, while a third female exhibited an enlarged uterus. A pregnant female from Valle de Cauca was recorded as carrying one fetus in mid-April; two juveniles were collected in late July.

The structural reinforcement associated with the robustness of the upper and lower inner incisor teeth in $S$. koopmanhilli may be adaptive for the frugivorous exploitation of the rich flora diversity in the Choco region. Similarly, the spacing between the molariform teeth is less obvious but both suggest specialized food habits.

Specimens Examined.-Sturnira mordax. COSTA RICA. Alajuela: $4.2 \mathrm{~km}$ SE Cariblanco, $1100 \mathrm{~m}, 12$ February 1983, T.J. McCarthy, 1 O’, 2 ᄋ (CM). Heredia: $1.0 \mathrm{~km} \mathrm{S,} 11.5 \mathrm{~km}$ E San Miguel, Río Cascante, 680 m, 3, 4, 6, 11 April 1986, R.K. LaVal, D.E. Wilson, 3 o", 2 ᄋ (USNM); 3.5 km S, 11.5 km E San Miguel, Río San Rafael, 1000 m, 6, 9, 13 April 1986, R.K. LaVal, D.E. Wilson, 2 o', 7 ㅇ (USNM); 4 km E Vara Blanca, 2050 m, 11 April 1986, D.E. Wilson, 1 ㅇ (USM); 5 km E Vara Blanca, 2050 m, 17, 18 April 1986, D.E. Wilson, 1 O’, 1 ㅇ (USNM). San José: La Palma, 630 m, 4 August 1961, A. Starrett, 1 q (USNM).

Sturnira mordax. PANAMA. Bocas del Torro: "Fish Camp," 1607 m, 24, 25, 27 March 1976, R.K. Enders, 2 o", 3 \%, (USNM); "First Water" (=Upper Camp, 1975), 2140 m, 28-31 March 1976, R.K. Enders, 4 O', 7 o (USNM); Cerro Colorado, Quebrada Alicia 25 [30] km NNE San Felix (Chiriquí), 1475 m, 13 June 1980, R.J. Izor, R.H. Pine, 2 ○ (USNM); Quebrada de los Gatos, $3 \mathrm{~km}$ NE Escopeta Camp [not located], 16 June 1980, R. H. Pine, 1 q (USNM). Chiriquí: Finca Santa Clara, 14.5 [17.5] km NW El Volcán, 1200 m, [no or questionable dates], R.K. Enders, 2 o', 2 o (USNM). Fortuna Dam Site, Río Chiriquí, 20 km ESE Boquete, 1000 m, October 1976, collector unknown, 1 Ơ (USNM); 6.4 km S El Volcán, 1100 m, 25-26 April 1962, E.T. Tyson, 4 ơ, 3 ㅇ (USNM); 1.6 km E Cuesta de Piedra, 850 m, 2-5 April 1962, E.T. Tyson, 4 ơ, 5 ㅇ (USNM).

Sturnira ludovici. ECUADOR. Carchi: El Pailón, at Río Pailón, 970 m, 19-21 December 1991, I. Manzano, T.J. McCarthy, 1 ơ, 4 o (CM,2;UMMZ,3); Praderas del Miravalle, 779 m, 20 August 1984, L. Albuja V., P. Mena V., 1 o, (EPN); Chimborazo: Planchas, 2900 m, 13 October 1976, P. O’Neill, 1 o (EPN). Esmeraldas: Cachabí, 300 m, 9 August 1935, C. Olalla, 1 O' (MNHN); Alto Tambo, Hda. La Granada, 570 m, 14, 16 August 1984, L. Albuja V., P. Mena V., 2 o (EPN); Cristal, 950 m, 30 December 1991-1 January 1992, I. Manzano, T.J. McCarthy, 3 Ơ, 2 o (CM); Reserva Ecológica Cotacachi-Cayapas, Cordillera de Toisán, Los Pambiles at Río las Piedras, 1200 m, 24 July 1985, P. Mena V., J. Regalado, 23-27 November 1991, I. Manzano, T.J. McCarthy, 6 ơ, 4 ○ (CM,9;EPN,1). Pichincha: El Paraiso Villaflora, 1321 m, 12 December 1984, L. Albuja V., P. Mena V., 1 ㅇ, (EPN); Gualea, 1220 m, April, February 1924, L. Soderstrom. 3 O' (AMNH,2;MCZ,1); Reserva Forestal ENDESA, Puerto Quito, 700 m, 27 December 1985, L. Albuja V., P. Mena V., 1 ○ (EPN); Mindo, 1264 m, 24 June 1913, G. Hammond, 1 ơ (FMNH). 


\section{ACKNOWLEDGMENTS}

We are grateful for the Ecuadorean bat collecting efforts of P. Mena V. and J. Regalado B. in July 1985 and I. Manzano in November 1991 at Los Pambiles. Trips to Los Pambiles were guided and assisted by F. Angulo and family, Cuellaje. Field work in 1985 was supported by the Primate Action Fund, World Wildlife Fund, which was administered by R. Mittermeier for the study of Ateles fusciceps. Field support in 1991 was provided by the Edward O'Neil Field Fund, Carnegie Museum of Natural History. Respective permits were granted by A. Paucar and $\mathrm{H}$. Cabrera A., Departamento de Areas Naturales y Vida Silvestre, Ministerio del Ambiente, Ecuador. We thank the assistance, collaboration, and coordination in the overall field project from S. Añaña (Federación Chachi), J.M. Jaramillo B. (Patrimonio Cultura de la Casa de la Cultura Ecuatoriana), N. and J. Nazareno (San Miguel), G. Añapa (Río Grande de Cayapas), A. Añapa de Calle Mansa (Río San Miguel), A. Añapa del Salto (Río Bravo), V. Cevallos (Universidad Católica), and C. Madden, C. Welch, and R. Barriga (EPN). Instituto Nacional de Meteorología e Hidrología (INAMHI), Quito, provided unpublished rainfall data.

We acknowledge the contributions in Colombia of students and colleagues for their efforts and assistance in the field, including $\mathrm{J}$. Albornoz R., G. Cantillo, O. Ospino, V. Rojas D., and C.A. Saavedra. Special thanks to V. Rojas D. and his assistance with data for this paper and his efforts in the collections (UV) in Calí. The Departamento de Biología, UV, supported fieldwork with time and resources.

We thank the following curatorial staffs who allowed us permission to examine and study specimens, provide loans, and/or assistance in different ways: K.F. Koopman and G.G. Musser (AMNH); J.R. Wible, D.A. Schlitter, and S. McLaren, (CM); P. Mena V. (EPN); B.D. Patterson and W. Stanley (FMNH); M. Rutzmoser and J. Chupasco (MCZ); M. Trainier and J. Cuisin (MNHN); P. Myers (UMMZ); L. Gordon, R.D. Fisher, C.O. Handley, Jr., and A.L. Gardner (USNM); V. Rojas D. (UV). The hospitality of G.G. Musser (AMNH) is appreciated when we initiated our study of Sturnira. Partial travel expenses to study S. mordax in Calí and Paris were supported by J.O. Matson. Photographic needs were assisted by J. Mahlberg and J.R. Wible, besides the SEM photos by T. Harper, University of Pittsburgh; W. Mann, University of Pittsburgh assisted Colombian maps; G. Scanlon assisted with a figure. M.A. Schmidt produced the tables. A.L. Gardner, J. Arroyo-Cabrales, C. Iudica, and J.R. Wible critiqued and enhanced the manuscript. Completion of this project was possible because of courtesies by J.R. Wible.

\section{LITERATURE CITED}

Alberico, M. 1994. First record of Sturnira mordax from Colombia with range extensions for other bat species. Trianea, 5:335-341.

Alberico, M., AND E. Velasco. 1991. Description of a new broadfaced bat from Colombia. Bonner zoologische Beiträge, 42:237-239.

Alberico, M., A. Cadena, J. Hernández Camacho, and Y. MuñozSABA. 2000. Mammals (Synapsida: Theria) of Colombia. Biota Colombiana, 1:44-75.

Albuja V., L. 1999. Murciélagos del Ecuador. Segunda edición. Cicetrónic Cia, Quito, Ecuador.

Albuja V., L., AND A.L. Gardner. 2005. A new species of Lonchophylla Thomas (Chiroptera: Phyllostomidae) from Ecuador. Proceedings of the Biological Society of Washington, 118:442-449.

Contreras-Vega, M., and A. Cadena. 2000. Una nueva especie del genero Sturnira (Chiroptera: Phyllostomidae) de los Andes Colombianos. Revista de la Academia Colombiana de Ciencias Exactas, Físicas y Naturales, 26:285-287.

DÁvalos, L.M. 2004. A new Chocoan species of Lonchophylla (Chiroptera: Phyllostomidae). American Museum Novitates, 3426:1-14.
Davis, W.B. 1980. New Sturnira (Chiroptera: Phyllostomidae) from Central and South America, with key to currently recognized species. Occasional Papers of The Museum, Texas Tech University, $70: 1-5$.

Duellman, W.E. 1979. The herpetofauna of the Andes: patterns of distribution, origin, differentiation, and present communities. Pp. 371-459, in The South American Herpetofauna: Its Origin, Evolution, and Dispersal (W.E. Duellman, ed.). University of Kansas, Lawrence, Kansas.

GentRY, A.H. 1982. Phytogeographic patterns as evidence for a Chocó refuge. Pp. 112-136, in Biological Diversification in the Tropics (G.T. Prance, ed.). Columbia University Press, New York.

. 1986. Species richness and floristic composition of Chocó region plant communities. Caldasia, 15:71-91.

HAFFER, J. 1967. Speciation in Colombian forest birds west of the Andes. American Museum Novitates, 2294:1-57.

Handley, C.O., JR. 1966. Descriptions of new bats (Choeroniscus and Rhinophylla) from Colombia. Proceedings of the Biological Society of Washington, 79:85-88.

Hershkovitz, P. 1971. Basic crown patterns and cusp homologies of mammalian teeth. Pp. 95-150, in Dental Morphology and Evolution (A.A. Dahlberg, ed.). University of Chicago Press, Chicago.

IBAÑEZ, C. 1980. Descripción de una nuevo género de quiróptero neotropical de la familia Molossidae. Doñana, Acta Vertebrata, 7:104-111.

Koopman, K.F. 1994. Chiroptera: Systematics. Handbook of Zoology, Vol. VIII, pt. 60, Mammalia, 1-217.

LYNCH, J.D. 1971. Redescriptions of three little-known Eleutherodactylus from northwestern Ecuador (Amphibia: Leptodactylidae). Transactions of the Kansas Academy of Science, 73:169-180.

1980. Systematic status and distribution of some poorly known frogs of the genus Eleutherodactylus from the Chocoan lowlands of South America. Herpetologica, 36:175-189.

MadDen, R.H., AND L. Albuja V. 1989. Estado actual de Ateles fusciceps fusciceps en el noroccidente Ecuatoriano. Politécnica, 14:113-157.

McCARThy, T.J., L. Albuja V., AND I. Manzano. 2000. Rediscovery of the brown sac-wing bat, Balantiopteryx infusca (Thomas, 1897), in Ecuador. Journal of Mammalogy, 81:958-961.

McCarthy, T.J., J.O. Matson, B. Rodriguez H., Y C.O. Handley, JR. 2005. Distribution, morphometrics, and identification of the Talamancan epaulette bat (Sturnira mordax) of Costa Rica and Panama. Pp. 331-344, in Contribuciones mastozoológicas en homenaje a Bernardo Villa (V. Sánchez-Cordero y R. A. Medellín, eds.). Universidad Nacional Autónoma de México, México.

Pacheco, V., AND B.D. Patterson. 1991. Phylogenetic relationships of the New World bat genus Sturnira (Chiroptera:Phyllostomidae). Pp. 101-121, in Contributions to Mammalogy in Honor of Karl F. Koopman (T.A. Griffiths and D. Klingener, eds.). Bulletin of the American Museum of Natural History, 206.

SÁnchez-Hernández, C., L. Romero-Almaraz, and J. Cuisin. 2002. Sturnira mordax (Chiroptera,Phyllostomidae) in Ecuador. Mammalia, 66:439-441.

SÁnchez-Hernández, C., M.L. Romero-Almaraz, and G.D. Schnell. 2005. New species of Sturnira (Chiroptera: Phyllostomidae) from northern South America. Journal of Mammalogy, 86:866-872.

TAMSITT, J.R., AND C. HäUSER. 1985. Sturnira magna. Mammalian Species, 240:1-4.

Terborgh, J., AND B. Winter. 1982. Evolutionary circumstances of species with small ranges. Pp. 587-600, in Biological Diversification in the Tropics (G.T. Prance, ed.). Columbia University Press, New York. 
